Notfall Rettungsmed 2008 $11: 260-265$

DOI 10.1007/s10049-008-1057-1

Online publiziert: 23. Mai 2008

\section{Redaktion}

C.K. Lackner, München

M. Fischer, Göppingen

W. Schreiber, Wien
E. von Elm ${ }^{1,7}$ - D.G. Altman ${ }^{2}$ M. Egger, ${ }^{1,3}$. S.J. Pocock ${ }^{4}$ P.C. Gøtzsche ${ }^{5}$. J.P. Vandenbroucke ${ }^{6}$ für die STROBE-Initiative

${ }^{1}$ Institut für Sozial- und Präventivmedizin (ISPM), Universität Bern

${ }^{2}$ Centre for Statistics in Medicine, University of Oxford

${ }^{3}$ Department of Social Medicine, University of Bristol

${ }^{4}$ London School of Hygiene and Tropical Medicine, University of London

${ }^{5}$ Nordic Cochrane Centre, Copenhagen

${ }^{6}$ Department of Clinical Epidemiology, Leiden University Hospital, Leiden

${ }^{7}$ Institut für Medizinische Biometrie und Medizinische Informatik, Universitätsklinikum Freiburg

\title{
Das Strengthening the Reporting of Observational Studies in Epidemiology (STROBE-) Statement
}

\author{
Leitlinien für das Berichten von \\ Beobachtungsstudien
}

Für viele Fragen der medizinischen Forschung werden Beobachtungsstudien eingesetzt [10]. Ein Großteil der Forschung über Krankheitsursachen beruht auf Kohorten-, Fallkontroll- und Querschnittsstudien. Solche Beobachtungsstudien spielen außerdem bei der Erforschung von Nutzen und Schaden von medizinischen Interventionen eine Rolle [3]. Nicht alle Fragen bezüglich einer bestimmten Intervention können durch randomisierte Studien beantwortet werden. So sind Beobachtungsstudien zum Beispiel besser dafür geeignet, seltene oder spät auftretende Nebenwirkungen von Interventionen zu erkennen. Sie zeigen auch eher an, welche Wirkung in der täglichen medizinischen Praxis tatsächlich erreicht wird [19].

Es ist wichtig, dass über Forschung in transparenter Weise berichtet wird und der Leser so die Möglichkeit hat, nachzuvollziehen, was geplant, durchgeführt und herausgefunden wurde und welche Schlussfolgerungen gezogen wurden. Die Glaubwürdigkeit der Forschung hängt davon ab, dass Dritte die Stärken und Schwächen von Studiendesign, -durchführung und -auswertung kritisch beurteilen können. Transparentes Berichten ist auch notwendig, um entscheiden zu können, ob und welche Studienergebnisse in systematischen Übersichtsarbeiten berücksichtigt werden können [13,8].

Trotzdem fehlen in Publikationen über beobachtende Forschung oft wichtige Informationen, oder sie sind unklar. So ergab eine Analyse von epidemiologischen Studien, die in allgemeinen und spezialisierten medizinischen Fachzeitschriften veröffentlicht wurden, dass die Auswahl von potenziellen Confoundern oft nicht begründet wurde [22]. Auch erläuterten nur wenige Berichte über psychiatrische Fallkontrollstudien die Methoden, die zur Identifikation von Fällen und Kontrollen angewendet wurden [14]. Und in einer Erhebung von longitudinalen Studien in der Schlaganfallforschung wurden in 17 von 49 Artikeln (35\%) die Einschlusskriterien nicht erläutert [26]. Es wurde bereits argumentiert, dass wegen fehlender Klarheit in der Berichterstattung die Ergebnisse der Forschung nur verzögert genutzt werden könnten [4] und dass Bedarf nach einer Leitlinie für das Berichten von Beobachtungsstudien besteht $[1,23]$.

Durch Empfehlungen für das Berichten von Forschungsergebnissen kann die
Qualität der Artikel verbessert werden. Das Consolidated Standards of Reporting Trials (CONSORT-) Statement wurde 1996 erarbeitet und 5 Jahre später revidiert [17]. Diese Initiative wurde von vielen medizinischen Fachzeitschriften unterstützt [15]. Dies half, die Qualität der Berichte über randomisierte Studien zu verbessern [21, 7]. Ähnliche Initiativen für andere Forschungsgebiete folgten, wie zum Beispiel für Metaanalysen von randomisierten Studien [16] und für diagnostische Studien [5]. Um Empfehlungen für das Berichten von Beobachtungsstudien zu erarbeiten, hat ein Netzwerk von Methodikern, Forschern und Herausgebern wissenschaftlicher Zeitschriften das Strengthening the Reporting of Observational Studies in Epidemiology (STROBE) Statement geschaffen.

\section{Ziele und Anwendung des STROBE-Statements}

Das STROBE-Statement besteht aus einer Checkliste, deren Punkte in Artikeln über Kohorten-, Fallkontroll- oder Querschnittsstudien (den 3 Hauptstudiendesigns in der analytischen Epidemiolo- 
gie) behandelt werden sollten. Damit soll lediglich eine Hilfestellung gegeben werden, wie beobachtende Forschung gut berichtet werden kann. Die Empfehlungen sollen jedoch keine Vorschriften für den Entwurf oder die Durchführung von Studien sein. Obwohl die Bewertung von Studien eine klare Darstellung voraussetzt, soll diese Checkliste kein Maßstab zur Bewertung der Qualität beobachtender Forschung sein.

Wir stellen hier das STROBE-Statement vor und erläutern dessen Entwicklung. In einem detaillierten Begleitartikel (Explanation and Elaboration; [27, 28, 29]) begründen wir die Aufnahme der einzelnen Punkte in die Checkliste. Außerdem erläutern wir den methodologischen Hintergrund und geben Beispiele dafür, was wir unter transparentem Berichten verstehen. Wir empfehlen dringend, die STROBE-Checkliste in Verbindung mit diesem erläuternden Begleitartikel zu gebrauchen. Er ist frei auf den folgenden Webseiten zugänglich:

\section{- PLoS Medicine}

(http://www.plosmedicine.org),

- Annals of Internal Medicine

(http://www.annals.org),

- Epidemiology

(http://www.epidem.com).

\section{Entwicklung des STROBE-Statements}

Im Jahr 2004 gründeten wir die STROBE-Initiative, sicherten die Finanzierung eines Workshops und richteten eine Webseite ein (http://www.strobestatement.org). Wir suchten in Lehrbüchern, bibliographischen Datenbanken, Literaturverzeichnissen und persönlichen Dateien nach relevanter Literatur, wie z. B. früheren Empfehlungen, empirischen Studien über Forschungsberichte und Artikeln über relevante methodologische Forschung. Da die beobachtende Forschung mit vielen verschiedenen Studiendesigns arbeitet, erschien es uns notwendig, den Anwendungsbereich von STROBE frühzeitig festzulegen. Wir konzentrierten uns auf die 3 Studiendesigns, die in der analytischen beobachtenden Forschung am häufigsten eingesetzt werden: Kohorten-, Fallkontroll- und Querschnittsstudien.

Notfall Rettungsmed 2008 · 11:260-265 DOI 10.1007/s10049-008-1057-1

E. von Elm · D.G. Altman · M. Egger · S.J. Pocock · P.C. Gøtzsche · J.P. Vandenbroucke für die STROBE-Initiative

Das Strengthening the Reporting of Observational

Studies in Epidemiology (STROBE-) Statement. Leitlinien

für das Berichten von Beobachtungsstudien

\section{Zusammenfassung}

Ein Großteil der biomedizinischen Forschung ist beobachtend, und die Qualität der veröffentlichten Berichte über diese Forschung ist oft unzureichend. Dies behindert die Beurteilung der Stärken und Schwächen einer Studie und ihrer Übertragbarkeit. Die Strengthening the Reporting of Observational Studies in Epidemiology (STROBE-) Initiative hat Empfehlungen entwickelt, was in einem akkuraten und vollständigen Bericht einer Beobachtungsstudie enthalten sein sollte. Die Empfehlungen wurden von uns so definiert, dass sie 3 Hauptstudientypen abdecken: Kohorten-, Fallkontroll- und Querschnittsstudien. Im September 2004 veranstalteten wir einen zweitägigen Workshop mit Methodikern, Forschern und Herausgebern wissenschaftlicher Zeitschriften, um eine Checkliste zu entwerfen. Anschließend wurde der Entwurf bei mehreren Treffen der Koordinierungsgruppe und nach E-Mail-Diskussionen mit der erweiterten STROBE-Gruppe revidiert und dabei empirische Evidenz und methodologische Aspekte berücksich-

\section{The Strengthening the Reporting of Observational Studies in Epidemiology (STROBE) Statement: Guidelines for reporting of observational studies}

\section{Abstract}

Much of biomedical research is observational. The reporting of such research is often inadequate, which hampers the assessment of its strengths and weaknesses and of a study's generalizability. The Strengthening the Reporting of Observational Studies in Epidemiology (STROBE) Initiative developed recommendations on what should be included in an accurate and complete report of an observational study. We defined the scope of the recommendations to cover three main study designs: cohort, case-control, and cross-sectional studies. We convened a 2-day workshop in September 2004, with methodologists, researchers, and journal editors to draft a checklist of items. This list was subsequently revised during several meetings of the coordinating group and in e-mail discussions with the larger group of STROBE contributors, taking into account empirical evidence and methodological considerations. tigt. Das Ergebnis des Workshops und des anschließenden iterativen Prozesses aus Beratung und Revision war eine Checkliste von 22 Punkten (STROBE-Statement), die sich auf die Bereiche Titel, Abstract, Einleitung, Methoden, Ergebnisse und Diskussion eines Artikels beziehen. 18 der Punkte sind relevant für alle 3 Studiendesigns, während 4 der Punkte spezifisch für Kohorten-, Fallkontrollund Querschnittsstudien sind. Ein ausführlicher Begleitartikel (Explanation and Elaboration) wurde separat veröffentlicht und ist auf den Webseiten von PLoS Medicine, Annals of Internal Medicine und Epidemiology frei zugänglich. Wir hoffen, dass das STROBE-Statement dazu beitragen kann, dass Beobachtungsstudien besser berichtet werden.

\section{Schlüsselwörter}

Beobachtungsstudien · Kohortenstudien . Fallkontrollstudien · Querschnittsstudien . Medizinische Forschung 


\section{Originalien}

Tab. 1 Das STROBE-Statement - Checkliste der Punkte, die in Berichten über Beobachtungsstudien enthalten sein sollten

Nr. Empfehlung

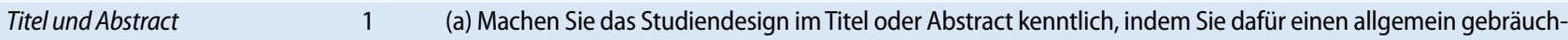
lichen Begriff verwenden

(b) Verfassen Sie für das Abstract eine aussagefähige und ausgewogene Zusammenfassung dessen, was in der Studie gemacht wurde und was herausgefunden wurde

\section{Einleitung}

Hintergrund/Rationale

Zielsetzungen

Methoden

Studiendesign

Rahmen

Studienteilnehmer

Variablen

Datenquellen/Messmethoden

Bias

Studiengröße

Quantitative Variablen

Statistische Methoden

Ergebnisse

Teilnehmer

Deskriptive Daten (Give Wenn nötig, beschreiben Sie, wie Kategorien (Gruppierungen) gebildet wurden und warum

(a) Beschreiben Sie alle statistischen Methoden, einschließlich der Methoden, die für die Kontrolle von Confounding verwendet wurden

(b) Beschreiben Sie Verfahren, mit denen Subgruppen und Interaktionen untersucht wurden

(c) Erklären Sie, wie mit fehlenden Daten umgegangen wurde

(d) Kohortenstudie - Erklären Sie gegebenenfalls wie mit dem Problem des vorzeitigen Ausscheidens aus der Studie („loss to follow-up") umgegangen wurde

Fallkontrollstudie - Beschreiben Sie gegebenenfalls wie das Matching (Paarbildung) von Fällen und Kontrollen bei der Auswertung berücksichtigt wurde

Querschnittsstudie - Beschreiben Sie gegebenenfalls die Auswertungsmethoden, die die gewählte Strategie zur Stichprobenauswahl (Sampling strategy) berücksichtigen

(e) Beschreiben Sie vorgenommene Sensitivitätsanalysen

(a) Geben Sie die Anzahl der Teilnehmer während jeder Studienphase an, z. B. die Anzahl der Teilnehmer, die potenziell geeignet waren, die auf Eignung untersucht wurden, die als geeignet bestätigt wurden, die tatsächlich an der Studie teilgenommen haben, deren Nachbeobachtung abgeschlossen wurde und deren Daten ausgewertet wurden

(b) Geben Sie die Gründe für die Nicht-Teilnahme in jeder Studienphase an

(c) Erwägen Sie die Darstellung in einem Flussdiagramm

14* (a) Beschreiben Sie Charakteristika der Studienteilnehmer (z. B. demographische, klinische und soziale Merkmale) sowie Expositionen und mögliche Confounder

(b) Geben Sie für jede Variable die Anzahl der Teilnehmer mit fehlenden Daten an

(c) Kohortenstudie - Fassen Sie die Nachbeobachtungszeit zusammen (z. B. Mittelwert und Gesamtzeitraum) 


\begin{tabular}{|c|c|c|}
\hline Ergebnisdaten & $15^{*}$ & $\begin{array}{l}\text { Kohortenstudie - Berichten Sie über die Anzahl der Zielereignisse oder statistische Maßzahlen (z. B. Mittelwert } \\
\text { und Standardabweichung) im zeitlichen Verlauf } \\
\text { Fallkontrollstudie - Berichten Sie über Teilnehmerzahlen in jeder Expositionskategorie oder über statistische } \\
\text { Maßzahlen der Exposition (z. B. Mittelwert und Standardabweichung) } \\
\text { Querschnittstudie - Berichten Sie über die Anzahl der Zielereignisse oder statistische Maßzahlen (z. B. Mittel- } \\
\text { wert und Standardabweichung) }\end{array}$ \\
\hline \multirow[t]{3}{*}{ Hauptergebnisse } & \multirow[t]{3}{*}{16} & $\begin{array}{l}\text { (a) Geben Sie die unadjustierten Schätzwerte an und gegebenenfalls auch die Schätzwerte, in denen Adjus- } \\
\text { tierungen für die Confounder vorgenommen wurden sowie deren Präzision (z. B. 95\%-Konfidenzintervall); } \\
\text { machen Sie deutlich, für welche Confounder adjustiert wurde und warum diese berücksichtigt wurden }\end{array}$ \\
\hline & & $\begin{array}{l}\text { (b) Wenn stetige Variablen kategorisiert wurden, geben Sie die oberen und unteren Grenzwerte der einzelnen } \\
\text { Kategorien an }\end{array}$ \\
\hline & & $\begin{array}{l}\text { (c) Wenn relevant, erwägen Sie, für aussagekräftige Zeiträume Schätzwerte relativer Risiken auch als absolute } \\
\text { Risiken auszudrücken }\end{array}$ \\
\hline Weitere Auswertungen & 17 & $\begin{array}{l}\text { Berichten Sie über weitere vorgenommene Auswertungen, z. B. die Analyse von Subgruppen und Wechselwir- } \\
\text { kungen (Interaktionen) sowie Sensitivitätsanalysen }\end{array}$ \\
\hline \multicolumn{3}{|l|}{ Diskussion } \\
\hline Hauptergebnisse & 18 & Fassen Sie die wichtigsten Ergebnisse in Hinsicht auf die Studienziele zusammen \\
\hline Einschränkungen & 19 & $\begin{array}{l}\text { Diskutieren Sie die Einschränkungen der Studie und berücksichtigen Sie dabei die Gründe für möglichen Bias } \\
\text { oder Impräzision } \\
\text { Diskutieren Sie die Richtung sowie das Ausmaß jedes möglichen Bias }\end{array}$ \\
\hline Interpretation & 20 & $\begin{array}{l}\text { Nehmen Sie eine vorsichtige übergreifende Interpretation der Resultate vor und berücksichtigen Sie dabei } \\
\text { die Ziele und Einschränkungen der Studie, die Multiplizität der Analysen, die Ergebnisse anderer Studien und } \\
\text { andere relevante Evidenz }\end{array}$ \\
\hline Übertragbarkeit & 21 & Besprechen Sie die Übertragbarkeit (externe Validität) der Studienergebnisse \\
\hline \multicolumn{3}{|l|}{ Zusätzliche Informationen } \\
\hline Finanzierung & 22 & $\begin{array}{l}\text { Geben Sie an, wie die vorliegende Studie finanziert wurde, und erläutern Sie die Rolle der Geldgeber. Machen } \\
\text { Sie diese Angaben gegebenenfalls auch für die Originalstudie, auf welcher der vorliegende Artikel basiert }\end{array}$ \\
\hline \multicolumn{3}{|c|}{$\begin{array}{l}\text { * Geben Sie diese Informationen jeweils gesondert für Fälle und Kontrollen in Fallkontrollstudien an, und gegebenenfalls für exponierte und nicht-exponierte Gruppen in } \\
\text { Kohorten- und Querschnittsstudien. } \\
\text { Anmerkung: Jeder Punkt der Checkliste wird in einem Begleitartikel (Explanation and Elaboration) besprochen, der methodologische Hintergründe diskutiert und } \\
\text { publizierte Beispiele transparenter Berichterstattung vorstellt. Die STROBE-Checkliste wird am besten zusammen mit diesem Artikel angewandt (frei zugänglich auf den fol- } \\
\text { genden Webseiten:PLoS Medicine http://www.plosmedicine.org, Annals of Internal Medicine http://www.annals.org und Epidemiology http://www.epidem.com). Zusätzliche } \\
\text { Versionen der Checkliste spezifisch für Kohorten-, Fallkontroll- oder Querschnittsstudien sind auf der STROBE-Webseite (http://www.Strobe-statement.org) auf Englisch } \\
\text { verfügbar. }\end{array}$} \\
\hline
\end{tabular}

Im September 2004 organisierten wir einen zweitägigen Workshop in Bristol (UK), an dem 23 Personen teilnahmen. Darunter waren Herausgeber und Mitarbeiter der Zeitschriften Annals of Internal Medicine, BMJ, Bulletin of the World Health Organization, International Journal of Epidemiology, JAMA, Preventive Medicine und The Lancet sowie Epidemiologen, Methodiker, Statistiker und Kliniker aus Europa und Nordamerika. 10 weitere an STROBE interessierte Personen, die am Workshop nicht teilnehmen konnten, gaben schriftliche Beiträge ab. In 3 Arbeitsgruppen wurden Punkte identifiziert, welche in spezifische Checklisten für jeden der 3 Studientypen aufgenommen werden sollten. Als Diskussionsgrundlage wurde eine vorher erstellte Liste verwendet, die auf unserer Webseite verfügbar ist. Die 3 Entwürfe wurden dann von allen Teilnehmern gemeinsam diskutiert. Wenn möglich, wurden einzelne Punkte dahingehend revidiert, dass sie für alle 3 Studiendesigns anwendbar wurden. In einer abschließenden Plenarsitzung beschloss die Gruppe eine Strategie zur Fertigstellung und Verbreitung des STROBEStatements.

Nach dem Workshop entwarfen wir eine kombinierte Checkliste, die alle 3 Studiendesigns umfasste, und veröffentlichten diese auf unserer Webseite. Die Teilnehmer des Workshops sowie andere Wissenschaftler und Herausgeber wurden eingeladen, diesen Entwurf zu kommentieren. In der Folge veröffentlichten wir auf unserer Webseite 3 revidierte Versionen und 2 Zusammenfassungen der erhaltenen Kommentare und der vorgenommenen Änderungen. Während dieser Zeit kam die Koordinierungsgruppe (die Autoren dieses Artikels) 8-mal für ein- bis zweitägige Treffen zusammen und hielt mehrere Telefonkonferenzen ab, um die Checkliste zu überarbeiten und um an dem vorliegenden Artikel und dem Begleitartikel (Explanation and Elaboration) zu arbeiten $[27,28,29]$. Die Koordinierungsgruppe lud 3 zusätzliche Mitautoren mit methodologischer und redaktioneller Erfahrung ein, die halfen, den Begleitartikel zu schreiben. Außerdem wurde das Feedback von mehr als 30 Personen eingearbeitet, deren Namen in Infobox 1 aufgeführt sind. Für Kommentare zu allen Entwürfen der Artikel wurden jeweils mehrere Wochen Zeit eingeräumt und die Teilnehmer per E-Mail an die Abgabetermine erinnert.

\section{Die STROBE-Komponenten}

Das STROBE-Statement besteht aus einer Checkliste von 22 Punkten, die unseres Erachtens essenziell für ein gutes $\mathrm{Be}$ - 
Infobox 1 Mitwirkende in der STROBE-Initiative

Die folgenden Personen haben zum Inhalt und zur Ausarbeitung des STROBE-Statement beigetragen: Douglas G. Altman, Maria Blettner, Paolo Boffetta, Hermann Brenner, Geneviève Chêne, Cyrus Cooper, George Davey-Smith, Erik von Elm, Matthias Egger, France Gagnon, Peter C. Gøtzsche, Philip Greenland, Sander Greenland, Claire InfanteRivard, John loannidis, Astrid James, Giselle Jones, Bruno Ledergerber, Julian Little, Margaret May, David Moher, Hooman Momen, Alfredo Morabia, Hal Morgenstern, Cynthia D. Mulrow, Fred Paccaud, Stuart J. Pocock, Charles Poole, Drummond Rennie, Martin Röösli, Dietrich Rothenbacher, Kenneth Rothman, Caroline Sabin, Willi Sauerbrei, Lale Say, James J. Schlesselman, Jonathan Sterne, Holly Syddall, Jan P. Vandenbroucke, Ian White, Susan Wieland, Hywel Williams, Guang Yong Zou.

richten von Beobachtungsstudien sind (- Tab. 1). Diese Punkte beziehen sich auf die folgenden Bereiche eines Artikels:

- Titel und Abstract (Punkt 1),

- Einleitung (Punkte 2 und 3),

- Methoden (Punkte 4-12),

- Ergebnisse (Punkte 13-17),

- Diskussion (Punkte 18-21),

- zusätzliche Informationen (Punkt 22 zur Finanzierung).

18 der Punkte sind auf alle 3 Studiendesigns anwendbar, während 4 Punkte (Punkte 6, 12, 14 und 15) jeweils spezifisch für ein Design sind, d. h. sich im Wortlaut ganz oder teilweise unterscheiden. Für einige der Punkte (mit Stern gekennzeichnet) sollten Angaben zu den Fällen und Kontrollen in Fallkontrollstudien beziehungsweise zu den Gruppen exponierter und nicht-exponierter Studienteilnehmer in Kohorten- und Querschnittsstudien getrennt gemacht werden. Zusätzlich zu der hier vorgestellten kombinierten Checkliste sind auf der STROBE-Webseite auch einzelne Checklisten (auf Englisch) für jedes der 3 Studiendesigns verfügbar.

\section{Schlussfolgerungen und Einschränkungen}

Das STROBE-Statement wurde entwickelt, um Autoren beim Verfassen von Berichten über analytische Beobachtungsstudien zu helfen, um Herausgebern wissenschaftlicher Zeitschriften und Gut- achter bei der Auswahl von Artikeln zur Publikation zu unterstützen und um die Leser veröffentlichter Studien bei deren kritischen Beurteilung zu helfen. Die Checkliste wurde in einem offenen Verfahren entwickelt, in das Erfahrungen aus früheren Initiativen, insbesondere CONSORT, einflossen. Wir sichteten relevante empirische Evidenz und methodologische Arbeiten und berieten über die aufeinander folgenden Entwürfe in einem umfassenden iterativen Verfahren. Daher basiert die hier vorgestellte Checkliste auf der Mitarbeit einer großen Anzahl von Personen mit verschiedenen Hintergründen und Perspektiven. Dieser Beratungsprozess erwies sich auch als sehr nützlich beim Verfassen des Begleitartikels (Explanation and Exploration; [27, 28, 29]), der in Verbindung mit der Checkliste genutzt werden sollte.

Beobachtungsstudien werden für vielfältige Zwecke eingesetzt, welche in einem Kontinuum von der Entdeckung neuer $\mathrm{Zu}$ sammenhänge bis hin zur Bestätigung oder Widerlegung früherer Erkenntnisse reichen $[27,28,29]$. Manche Studien sind hauptsächlich explorativer Natur und darauf ausgerichtet, interessante Hypothesen aufzustellen, während andere Studien darauf abzielen, klar definierten Hypothesen mithilfe bereits vorhandener Daten nachzugehen. Und in wieder einer anderen Art von Studien wird das Sammeln von neuen Daten sorgfältig aufgrund einer existierenden Hypothese geplant. Wir sind der Meinung, dass die hier vorgestellte Checkliste für jede dieser Studienarten von Nutzen ist.

Die Leser sollten immer verstehen können, was (und was nicht) in einer Studie geplant war, was durchgeführt und herausgefunden wurde und was die Ergebnisse bedeuten. Wir räumen ein, dass die Anwendung von STROBE derzeit auf nur 3 wichtige Typen von Beobachtungsstudien beschränkt ist. Wir würden Ergänzungen begrüßen, welche die Checkliste auch für andere Studiendesigns - wie z. B. Case-Crossover-Studien oder ökologische Studien - sowie für spezifische Fachgebiete anwendbar machten. Für das CONSORT-Statement sind inzwischen 4 Erweiterungen verfügbar [11, 6, 20, 9]. Eine erste Ergänzung des STROBE-Statements für genetische Assoziationsstudien ist in Arbeit: die STROBE Extension to Ge- netic Association studies (STREGA-) Initiative [12]. Um doppelten Aufwand zu vermeiden, bitten wir alle, die Ergänzungen für das STROBE-Statement entwickeln wollen, vorher die Koordinierungsgruppe zu kontaktieren.

Das STROBE-Statement sollte nicht als Versuch verstanden werden, ein starres Format für das Berichten von beobachtender Forschung festlegen zu wollen. Die einzelnen Punkte der Checkliste sollten in einem Artikel in ausreichender und klarer Form behandelt werden. Reihenfolge und Format hängen aber von den Vorlieben des Autors, dem Stil der Zeitschrift und den Gepflogenheiten des Forschungsgebiets ab. So behandeln wir zum Beispiel das Berichten von Ergebnissen in der Checkliste verteilt auf mehrere Punkte, wissen jedoch, dass Autoren diese Punkte möglicherweise in einem einzelnen Absatz oder in einer Tabelle besprechen. Ebenso könnte Punkt 22 der Checkliste, der die Finanzierung und die Rolle des Geldgebers betrifft, sowohl in einem Anhang als auch im Methodenteil des Artikels besprochen werden.

Es ist nicht unser Ziel, das Berichten zu standardisieren. Ein Herausgeber einer Fachzeitschrift forderte die Autoren randomisierter klinischer Studien auf, ihre Manuskripte vor dem Einreichen zu „CONSORTisieren“ [18]. Wir glauben nicht, dass Manuskripte „STROBEisiert“ werden sollten, im Sinne einer Regulierung des Stils oder der Terminologie. Vielmehr möchten wir die Autoren dazu ermutigen, erzählende Elemente einzuarbeiten, wie etwa die Beschreibung anschaulicher Fälle. Solche Elemente ergänzen die wesentlichen Informationen über eine Studie und verwandeln den Artikel in interessanten Lesestoff [25].

Wir möchten betonen, dass das STROBE-Statement nicht als Instrument zur Bewertung der Qualität von bereits veröffentlichten Ergebnissen beobachtender Forschung entwickelt wurde. Solche Instrumente wurden von anderen Gruppen entwickelt und in einer kürzlich veröffentlichten systematischen Übersichtsarbeit besprochen [24]. In unserem Begleitartikel verwendeten wir mehrere Studien als Beispiele, die zwar gut berichtet wurden, aber deren Ergebnisse in späteren Studien nicht bestätigt wur- 
den - das für uns entscheidende Merkmal war das gute Berichten, nicht die Frage, ob die Forschung an sich gut gemacht wurde. Wenn STROBE von Autoren und Zeitschriften in Zukunft benutzt wird, könnten Belange wie Confounding, Bias und die Übertragbarkeit von Studienergebnissen transparenter werden. Dies könnte dabei helfen, die übereifrige Berichterstattung über neue Ergebnisse innerhalb der Forschungsgemeinschaft und in der Boulevardpresse zu dämpfen [2] und die Studienmethodologie langfristig $\mathrm{zu}$ verbessern. Bessere Berichte könnten auch eine bessere Grundlage liefern, um $\mathrm{zu}$ entscheiden, wann neue Studien notwendig sind und was genau mit ihnen erforscht werden soll.

Wir haben nicht für jeden Punkt der Checkliste eine umfassende systematische Übersichtsarbeit erstellt und auch keine eigene Forschung betrieben, um eventuelle Lücken in der Evidenz zu schlieBen. Obwohl niemand von der Mitarbeit in diesem Projekt ausgeschlossen wurde, war die Zusammensetzung der Teilnehmergruppe doch von bereits existierenden Netzwerken beeinflusst. Die Gruppe war von Teilnehmern aus Europa und Nordamerika dominiert und somit geographisch nicht repräsentativ. Auch in Bezug auf Forschungsinteressen und -disziplinen war die Gruppe wahrscheinlich nicht repräsentativ. Wir möchten betonen, dass STROBE und andere Empfehlungen für das Berichten von Forschungsergebnissen als sich weiterentwickelnde Dokumente gesehen werden sollten, die kontinuierlich überprüft, verbessert und, wenn notwendig, verändert werden müssen. Vorschläge, wie STROBE weiter verbreitet werden kann, z. B. durch Publikation des vorliegenden Artikels in Fachzeitschriften und in anderen Sprachen, sind uns willkommen. Gruppen oder Einzelpersonen, welche die Checkliste in andere Sprachen übersetzen möchten, sollten sich bitte vorher mit der Koordinierungsgruppe beraten. Wir werden die Checkliste in Zukunft überarbeiten und dabei Kommentare, Kritik, neue Evidenz sowie Erfahrungswerte aus ihrer Nutzung berücksichtigen. Wir laden alle Leser ein, ihre Kommentare über die STROBEWebseite (http://www.strobe-statement. org) bei uns einzureichen.

\section{Korrespondenzadresse}

\section{Dr. E. von Elm}

Institut für Sozial- und Präventivmedizin (ISPM), Universität Bern

Finkenhubelweg 11, CH-3012 Bern

strobe@ispm.unibe.ch

Danksagung. Wir danken Gerd Antes, Kay Dickersin, Shah Ebrahim, und Richard Lilford für ihre Unterstützung der STROBE-Initiative. Wir danken den folgenden Institutionen, die Gastgeber der Arbeitstreffen der Koordinierungsgruppe waren: Institut für Sozial- und Präventivmedizin (ISPM), Universität Bern, Bern, Schweiz; Department of Social Medicine, University of Bristol, Bristol, UK; London School of Hygiene and Tropical Medicine, London, UK; Nordic Cochrane Centre, Copenhagen, Dänemark; und Centre for Statistics in Medicine, Oxford, UK. Wir danken 6 Gutachtern, die hilfreiche Kommentare zu einem früheren Entwurf dieses Artikels beigetragen haben.

Finanzierung. Der Workshop wurde von der European Science Foundation (ESF) finanziert. Zusätzliche Geldmittel wurden von der Medical Research Council Health Services Research Collaboration und dem National Health Services Research and Development Methodology Programme zur Verfügung gestellt.

Interessenkonflikt. Laut Erklärung der Autoren existieren keine Interessenkonflikte.

Für Informationen zur Weiterverwendung des Artikels s. STROBE-Webseite (http://www.strobe-statement.org). Die deutsche Übersetzung wurde von Caroline Mavergames und Erik von Elm erstellt. Sie basiert auf der englischen Originalversion des STROBE-Statements, die von den folgenden Zeitschriften veröffentlicht und frei zugänglich gemacht wurde: Annals of Internal Medicine, BMJ, Bulletin of the World Health Organization, Epidemiology, The Lancet, PLoS Medicine und Preventive Medicine.

\section{Literatur}

1. Anonymous (1981) Guidelines for documentation of epidemiologic studies. Epidemiology Work Group of the Interagency Regulatory Liaison Group. Am J Epidemiol 114: 609-613

2. Bartlett C, Sterne J, Egger M (2002) What is newsworthy? Longitudinal study of the reporting of medical research in two British newspapers. BMJ 325: 81-84

3. Black N (1996) Why we need observational studies to evaluate the effectiveness of health care. BMJ 312: 1215-1218

4. Bogardus ST, Concato J, Feinstein AR (1999) Clinical epidemiological quality in molecular genetic research: the need for methodological standards. JAMA 281: 1919-1926

5. Bossuyt PM, Reitsma JB, Bruns DE et al. (2003) Towards complete and accurate reporting of studies of diagnostic accuracy: The STARD Initiative. Ann Intern Med 138: 40-44

6. Campbell MK, Elbourne DR, Altman DG (2004) CONSORT statement: extension to cluster randomised trials. BMJ 328: 702-708

7. Egger $M$, Jüni $P$, Bartlett $C$ (2001) Value of flow diagrams in reports of randomized controlled trials. JAMA 285: 1996-1999

8. Egger M, Schneider M, Davey Smith G (1998) Spurious precision? Meta-analysis of observational studies. BMJ 316: 140-144
9. Gagnier JJ, Boon H, Rochon P et al. (2006) Reporting randomized, controlled trials of herbal interventions: an elaborated CONSORT statement. Ann Intern Med 144: 364-367

10. Glasziou P, Vandenbroucke JP, Chalmers I (2004) Assessing the quality of research. BMJ 328: 39-41

11. Ioannidis JP, Evans SJ, Gøtzsche PC et al. (2004) Better reporting of harms in randomized trials: an extension of the CONSORT statement. Ann Intern Med 141: 781-788

12. Ioannidis JP, Gwinn M, Little J et al. (2006) A road map for efficient and reliable human genome epidemiology. Nat Genet 38: 3-5

13. Jüni P, Altman DG, Egger M (2001) Systematic reviews in health care: assessing the quality of controlled clinical trials. BMJ 323: 42-46

14. Lee W, Bindman J, Ford T et al. (2007) Bias in psychiatric case-control studies: literature survey. $\mathrm{Br} J$ Psychiatry 190: 204-209

15. Moher D, Altman DG, Schulz KF, Elbourne DR (2004) Opportunities and challenges for improving the quality of reporting clinical research: CONSORT and beyond. CMAJ 171: 349-350

16. Moher D, Cook DJ, Eastwood S et al. (1999) Improving the quality of reports of meta-analyses of randomised controlled trials: the QUOROM statement. Quality of Reporting of Meta-analyses. Lancet 354: 1896-1900

17. Moher D, Schulz KF, Altman DG (2001) The CONSORT statement: revised recommendations for improving the quality of reports of parallel-group randomised trials. Lancet 357: 1191-1194

18. Ormerod AD (2001) CONSORT your submissions: an update for authors. Br J Dermatol 145: 378-379

19. Papanikolaou PN, Christidi GD, loannidis JP (2006) Comparison of evidence on harms of medical interventions in randomized and nonrandomized studies. CMAJ 174: 635-641

20. Piaggio G, Elbourne DR, Altman DG et al. (2006) Reporting of noninferiority and equivalence randomized trials: an extension of the CONSORT statement. JAMA 295: 1152-1160

21. Plint AC, Moher D, Morrison A et al. (2006) Does the CONSORT checklist improve the quality of reports of randomised controlled trials? A systematic review. Med J Aust 185: 263-267

22. Pocock SJ, Collier TJ, Dandreo KJ et al. (2004) Issues in the reporting of epidemiological studies: a survey of recent practice. BMJ 329: 883

23. Rennie $D$ (2001) CONSORT revised - improving the reporting of randomized trials. JAMA 285: 20062007

24. Sanderson S, Tatt ID, Higgins JP (2007) Tools for assessing quality and susceptibility to bias in observational studies in epidemiology: a systematic review and annotated bibliography. Int J Epidemiol 36: 666-676

25. Schriger DL (2005) Suggestions for improving the reporting of clinical research: the role of narrative. Ann Emerg Med 45: 437-443

26. Tooth L, Ware R, Bain C et al. (2005) Quality of reporting of observational longitudinal research. Am J Epidemiol 161: 280-288

27. Vandenbroucke JP, Elm E von, Altman DG et al. for the STROBE Initiative (2007) Strengthening the Reporting of Observational Studies in Epidemiology (STROBE): Explanation and Elaboration. PLoS Med 4: e297

28. Vandenbroucke JP, Elm E von, Altman DG et al. for the STROBE Initiative (2007) Strengthening the Reporting of Observational Studies in Epidemiology (STROBE): explanation and elaboration. Ann Intern Med 147:W163-W194

29. Vandenbroucke JP, Elm E von, Altman DG et al. for the STROBE Initiative (2007) Strengthening the Reporting of Observational Studies in Epidemiology (STROBE): explanation and elaboration. Epidemiology 18: 805-835 